\title{
An investigation into gender variation in the nutritional status of young adults
}

\author{
G. Glendinning, I. Alaunyte and F. Amirabdollahian \\ School of Health Sciences, Liverpool Hope University, Liverpool, L16 9JD
}

Previous research indicated that young adults often consume high quantity of convenience fast foods resulting in high dietary intake of total and saturated fats and minimal intake of fresh fruit and vegetables ${ }^{(1)}$. This has had a negative impact upon the health status of the young adults suggesting that they may be vulnerable to unhealthy dietary practices and positive energy intakes ${ }^{(2)}$. Previous studies have indicated controversial findings that there may be a gender variation in nutritional status of this population ${ }^{(3)}$; while average absolute nutrient intake for young men is often understandably higher than female counteracts, the studies often do not take into account the difference body size and composition and the impact of variation in energy intake and requirements of males and females. The purpose of this study was to assess the potential gender variation in nutritional status and quality of diet of young adults in residing in North West of England.

After securing ethical approval, 238 young adults aged 18-25 years were recruited in a cross-sectional study. Laboratory and physical measures of nutritional status such as biomarkers of fasting capillary blood glucose and lipid profile, weight, height, percentage body fat, BMI and blood pressure were assessed. Energy and macronutrient intake was measured using a validated 3-day diet diary and analysed using dietary assessment software Microdiet. Normal distribution was investigated using the Shapiro-Wilk test of normality and because the main variables were not normally distributed $(\mathrm{P}<0.05)$ and gender variation in nutritional status was investigated via nonparametric Mann Whitney U-Test using SPSS 22. Statistical significance was set at 0.05.

For females, the median percentage contributions of carbohydrate, fat, saturated fat and free sugar were significantly higher than males, whilst the median percentage contribution of protein was higher for males. Within fasting capillary blood lipid biomarkers, the median blood glucose concentration was significantly higher in males in comparison with females (Table 1).

Table 1. The median value for biomarkers and energy contribution from macronutrients between genders

\begin{tabular}{|c|c|c|c|c|c|c|c|}
\hline & \multicolumn{3}{|l|}{ Males } & \multicolumn{3}{|l|}{ Females } & \multirow[b]{3}{*}{ P-value } \\
\hline & \multirow[b]{2}{*}{ Median } & \multicolumn{2}{|c|}{ Quartiles } & \multirow[b]{2}{*}{ Median } & \multicolumn{2}{|c|}{ Quartiles } & \\
\hline & & $\overline{\mathrm{P} 25}$ & P75 & & $\overline{\mathrm{P}} 25$ & P75 & \\
\hline \multicolumn{8}{|l|}{ Percentage Contribution to Energy } \\
\hline $\mathrm{CHO}(\%)$ & $43 \cdot 1$ & $37 \cdot 8$ & $49 \cdot 2$ & $45 \cdot 7$ & $41 \cdot 7$ & $50 \cdot 4$ & $0 \cdot 018$ \\
\hline Protein (\%) & 19.8 & $16 \cdot 2$ & $24 \cdot 7$ & $16 \cdot 9$ & $14 \cdot 6$ & $19 \cdot 7$ & $0 \cdot 001$ \\
\hline Fat $(\%)$ & $33 \cdot 1$ & $28 \cdot 0$ & $37 \cdot 5$ & $35 \cdot 3$ & $29 \cdot 8$ & $38 \cdot 8$ & $0 \cdot 040$ \\
\hline$S F A(\%)$ & $10 \cdot 6$ & $8 \cdot 5$ & $13 \cdot 46$ & $12 \cdot 3$ & $10 \cdot 2$ & $15 \cdot 0$ & $0 \cdot 004$ \\
\hline$P U F A(\%)$ & $4 \cdot 8$ & $3 \cdot 5$ & $6 \cdot 30$ & $5 \cdot 0$ & $3 \cdot 6$ & $7 \cdot 2$ & $0 \cdot 329$ \\
\hline$M U F A(\%)$ & $10 \cdot 3$ & 8.5 & $12 \cdot 1$ & $10 \cdot 8$ & $8 \cdot 6$ & $12 \cdot 7$ & $0 \cdot 435$ \\
\hline Free sugar $(\%)$ & $14 \cdot 6$ & $9 \cdot 5$ & $19 \cdot 4$ & $17 \cdot 3$ & $13 \cdot 4$ & $21 \cdot 3$ & $0 \cdot 001$ \\
\hline \multicolumn{8}{|l|}{ Biomarkers } \\
\hline Whole blood Total Cholesterol (mmol/L) & 4.09 & $3 \cdot 42$ & 4.49 & $4 \cdot 10$ & $3 \cdot 52$ & $4 \cdot 45$ & 0.796 \\
\hline Whole blood Total HDL (mmol/L) & $1 \cdot 30$ & $1 \cdot 07$ & 1.50 & $1 \cdot 38$ & $1 \cdot 19$ & 1.64 & 0.039 \\
\hline Whole Blood Total TG ( mmol/L) & 0.86 & $0 \cdot 64$ & 1.42 & 1.00 & $0 \cdot 61$ & $1 \cdot 39$ & $0 \cdot 915$ \\
\hline Whole blood Total LDL (mmol/L) & $2 \cdot 23$ & 1.76 & 2.79 & $\mathbf{2} \cdot \mathbf{1 0}$ & 1.63 & $2 \cdot 57$ & $0 \cdot 229$ \\
\hline Whole Blood Glucose Concentration (mmol/L) & 4.96 & $4 \cdot 68$ & $5 \cdot 28$ & $4 \cdot 71$ & $4 \cdot 36$ & $5 \cdot 09$ & $0 \cdot 001$ \\
\hline
\end{tabular}

Higher contribution of total fat, SFA and free sugar to the energy intake of young females in comparison with males would warrants further investigation. This is particularly important as current levels of consumption are above the Dietary Reference Values and prolonged regular intake at this level may enhance the risk of non communicable diseases such as Type 2 diabetes and cardiovascular diseases.

1. Troiano RP, Briefel RR, Carroll MD, Bialostosky K. (2000) Am J Clin Nutr 72, 1343s-1353s

2. Hoffman DJ, Policastro P, Quick V, Lee SK (2006) J Am Coll Health 55, 41-5

3. Davy SR, Benes BA, Driskell JA. (2006) J Am Dietetic Association 106, 1673-1677 